\title{
Dos especies nuevas de Phlegmariurus Holub (Lycopodiaceae) para Costa Rica
}

\author{
Alexander Francisco Rojas-Alvarado y Rafael Rolando Calderón-Fallas \\ Universidad Nacional de Costa Rica. \\ Apdo. 86-3000, Heredia, Costa Rica.
}

Correspondencia

A. F. Rojas-Alvarado

e-mail: alfrojasa@yahoo.com

Recibido: 20 junio 2017

Aceptado: 11 septiembre 2017

Publicado on-line: diciembre 2017

\begin{abstract}
Resumen
Dos especies nuevas de Phlegmariurus Holub, $P$. gracilis A. Rojas y $P$. nanus A. Rojas \& R. Calderón, son descritas aquí. Ellas pertenecen al complejo $P$. reflexus. La primera se caracteriza por hojas en verticilos alternos de 4 o 5 y estas con el margen entero o dispersa y cortamente dentado. La segunda corresponde a un taxón con tallos bastante pequeños (6-11 cm de largo) y simples a 1-dicotomos, y las hojas con cilios largos y dispersos marginalmente.

Palabras clave: Huperzia, Lycopodiophyta, licofitos, nuevos taxones, Phlegmariurus.

Abstract

Two new species of Phlegmariurus Holub (Lycopodiaceae) from costa rica

Two new species of Phlegmariurus Holub, $P$. gracilis A. Rojas and $P$. nanus A. Rojas \& R. Calderón, are here described. They belong to the $P$. reflexus complex. The first is characterized by alternating leaves in whorls of 4 or 5 with entire or sparsely dentate margins. The second is distinguished by small (6-11 $\mathrm{cm}$ long) and simple to 1-dichotomous stems, and leaf margins sparsely longciliate.
\end{abstract}

Key words: Huperzia, Lycopodiophyta, lycophytes, new taxa, Phlegmariurus.

\section{Introducción}

Moran (2011), en su clave para Lycopodiopsida, indica como características de la familia Lycopodiaceae la homosporia, hojas no liguladas y raíces sin un canal aéreo central.

Lellinger (1989), asumió en su tratamiento para Costa Rica, Panamá y El Chocó sólo el reconocimiento de Lycopodium L. con los subgéneros: Cerniustachys Herter, Lycopodium y Selago Baker. Øllgaard (1995) utilizó para Mesoamérica la clasificación que divide a la familia en tres géneros: Huperzia Bernh., Lycopodiella Holub y Lycopodium $\mathrm{L}$.

Para Norteamérica, Wagner y Beitel (1992) utilizan Phlegmariurus Holub, Huperzia, Lycopodium, Diphasiastrum Holub,
Pseudolycopodiella Holub, Lycopodiella Holub y Palhinhaea Franco \& Vasc. dándole validez al trabajo realizado por Holub (1983). En contraste, para el Neotrópico, es hasta que Øllgaard (2012a y 2012b) hace las combinaciones de las especies a estos otros géneros reconocidos previamente.

Phlegmariurus fue acuñado por Holub (1964) en sustitución de Lycopodium sect. Phlegmariurus Herter, este último creado en 1909. Según la clave de géneros por Arana \& Øllgaard (2012), Phlegmariurus difiere de Huperzia por plantas sin bulbillos, esporas con lados convexos $0 \pm$ planos en el ecuador y ángulos obtusos o agudos.

Recientemente son pocos los aportes para Costa Rica que incluyen nuevos registros o taxones en Lycopodiaceae. Øllgaard (1993) publicó dos especies nuevas de Huperzia y 
combinó otro taxón, de estos dos corresponden actualmente a Phlegmariurus. Øllgaard (1995) registra 34 especies de Huperzia en Costa Rica, de las cuales tres son endémicas. Rojas (1996) registró una especie de Lycopodiella para este país. Rojas (2005) publicó una especie nueva de Huperzia y registró dos más para esta nación, de ellas la especie nueva y un registro corresponden actualmente a Phlegmariurus.

Øllgaard $(1988,1995)$ mencionó que Huperzia reflexa (Lam.) Trevis. (=Phlegmariurus reflexus (Lam.) B. Ø1lg.) es variable en caracteres como hoja y tamaño del tallo, dirección y agrupamiento de las hojas y margen de la hoja. Pero varias formas distintivas pueden estar creciendo entremezcladas en el mismo hábitat, indicando que existen diferencias genéticas.

Según la clave de Huperzia propuesta por Øllgaard (1995), H. reflexa difiere de $H$. eversa (Poir.) B. Øllg. por presentar vástagos generalmente 7-15 $\mathrm{mm}$ de ancho (incluyendo las hojas); hojas generalmente 4-8 $\mathrm{mm}$, en verticilos alternos de 7 u 8, linear-subuladas y rectas a marcadamente recurvadas. El autor también indica que es una especie neotropical y que junto con $\mathrm{H}$. eversa y $\mathrm{H}$. chamaleon forma un complejo que incluye cuatro o más elementos en Mesoamérica que requieren de más estudio.

El objetivo del presente trabajo es publicar bajo el estatus de especie a dos taxones previamente detectados por Øllgaard (1995) dentro del complejo $H$. reflexa (=Phlegmariurus reflexus (Lam.) B. Øllg.).

\section{Materiales y métodos}

En la presente investigación se describen dos especies nuevas de Phlegmariurus para Costa Rica, con base en el siguiente acervo bibliográfico de Lycopodiaceae neotropicales: Holub (1983), Lellinger (1989), Øllgaard (1987, 1988, 1992, 1993, 1995, 2012a y 2012b), Rojas (1996 y 2005). Además, se revisaron los especímenes del Herbario Nacional de Costa Rica (CR) y del herbario de la Universidad de Costa Rica (USJ).

\section{Resultados}

Phlegmariurus gracilis A. Rojas, sp. nov. (fig. 1). TIPO: COSTA RICA. Limón: Cordillera de Talamanca, Atlantic slope, $S$ side of unnamed cordillera between the Río Terbi and Río Siní, 2-4 airline $\mathrm{km} \mathrm{W}$ of the Costa Rican-Panamanian border, $9^{\circ} 09-11^{\prime} \mathrm{N}, 82^{\circ} 57-58^{\prime} \mathrm{W}, 2300-500 \mathrm{~m}, 11$ Sep 1984, G. Davidse et al. 28959 (holotipo: CR, 109070; isotipo: MO).

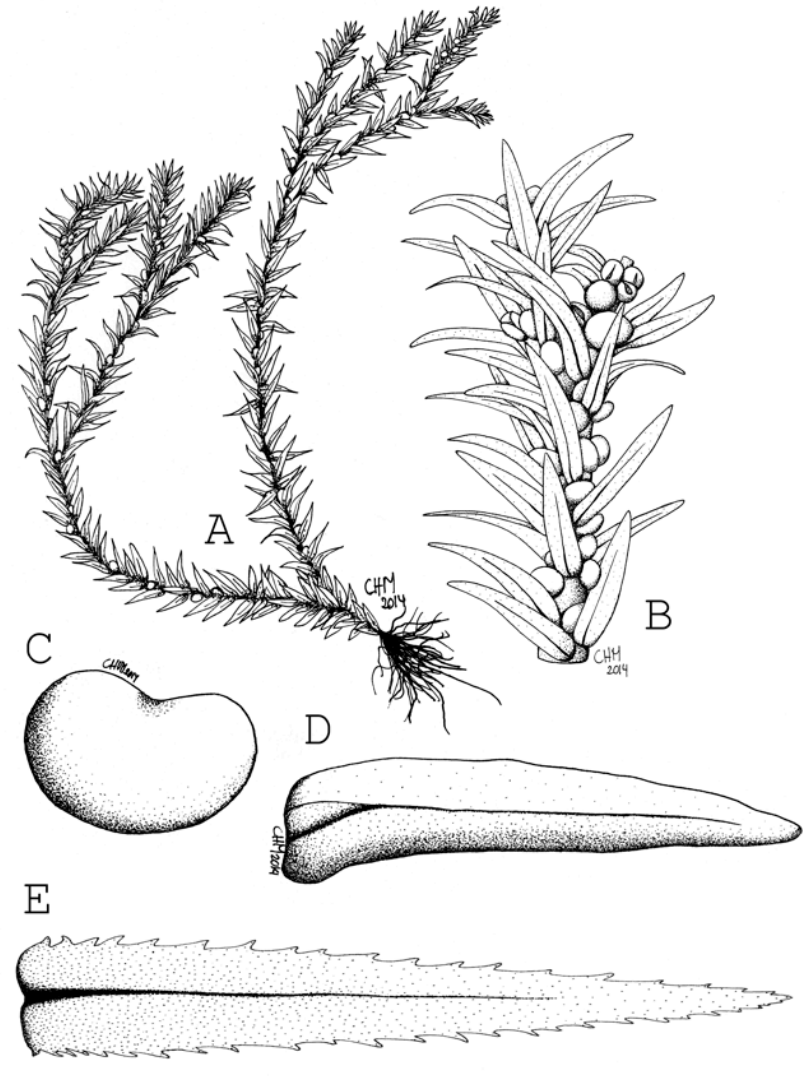

Figura 1: Dos especies parecidas de Phlegmariurus: A-D. P. gracilis (G. Davidse et al. 28959, CR). A) Hábito. B) Detalle de la planta. C) Esporangio. D) hoja. E. Hoja de $P$. reflexus (W. Haber \& W. Zuchowski 9074, CR). Figure 1: Two similar species of Phlegmariurus: $\boldsymbol{A}-\boldsymbol{D}$. P. gracilis (G. Davidse et al. 28959, CR). A) Habit. B) Plant detail. C) Sporangium. D) Leaf. E) Leaf of $P$. reflexus (W. Haber \& W. Zuchowski 9074, CR).

Diagnosis. Phlegmariurus gracilis differs from $P$. reflexus (Lam.) B. Ø1lg. by broader leaves [0.8-1.5 mm wide vs. 0.5-1.0 $(-1.2) \mathrm{mm}]$, these in whorls of 4 or 5 [vs. (6-) 7-8 (-9)] and with margins entire to sparsely and shortly dentate (vs. commonly densely dentate).

Descripción. Terrestres o epilíticas, a orillas de riachuelos y escorrentías; plantas $4,5-13 \mathrm{~cm}$ de largo, 2-3-dicotómicas, generalmente arqueadas, individuales o formando pequeñas poblaciones; vástagos 6-12 $\mathrm{mm}$ de ancho incluyendo las hojas, casi del mismo ancho en toda su extensión, homofilos; tallos 0,8-1,5 $\mathrm{mm}$ de diámetro (hasta $3 \mathrm{~mm}$ con esporangios), crestados por las bases decurrentes de las hojas; hojas 3-5 $\times$ ca. $1 \mathrm{~mm}$, dispuestas en verticilos irregulares alternos de 4-5, linear-lanceoladas, más anchas justo por encima de la base, levemente ascendentes (a veces reflexas cuando secas), rectas a recurvadas, 
herbáceas a cartáceas, el haz cóncavo cerca de la base y plano o convexo en la parte media y ápice, el envés aplanado o escasamente cóncavo a convexo, con una nervadura inconspicua, pálida abaxialmente (al menos en la base), los márgenes aplanados a levemente revolutos, enteros a corta y esparcidamente dentados, los dientes menos de $0,1 \mathrm{~mm}$ de largo, hialinos, ascendentes hasta en ángulo de $70^{\circ}$ con la costa; base de las hojas a menudo decurrente, ápice agudo; esporangios $1-1,5 \mathrm{~mm}$ de ancho.

Distribución. Costa Rica, conocida sólo de la Cordillera de Talamanca; bosques montanos, 2300-2900 m.

Etimología. El epíteto específico hace referencia a los tallos delgados con pocas hojas por verticilo.

Especímenes adicionales revisados. COSTA RICA. Cartago-San José: Cerro de La Muerte, entre Villa Mills y División, Carretera Panamericana Sur, 2900 m, 26 feb 1965, A. Jiménez 2980 (CR, 46428, F). San José: $\mathrm{S}$ of Cartago, ca. $1 \mathrm{~km}$ S of El Empalme along the Interamerican Hwy., 2400 m, 16 Jul 1970, D. Lellinger \& J. White 1202 (CR, 52138, US).

Phlegmariurus nanus A. Rojas \& R. Calderón, sp. nov. (fig. 2)

TIPO: COSTA RICA. Heredia: Heredia, Varablanca, orillas de la carretera rumbo a Sarapiquí, 1009'37'N, 8409'21'W, 1925 m, 29 set 2013, A. Rojas \& M. Obando 10517 (holotipo: CR; tres especímenes por distribuir).

Diagnosis. Phlegmariurus nanus differs from $P$. reflexus (Lam.) B. Øllg. by single (vs. clustered) stems, shorter, simple to 1-dichotomous (vs. 2-5dichotomous), and leaves longer (0.1-0.3 mm vs. $0.05-0.15 \mathrm{~mm}$ ) with margins sparsely ciliate (vs. dentate).

Descripción. Terrestres, completamente erectas, en individuos solitarios, pero comúnmente varias plantas en el mismo sitio; plantas $6-11 \mathrm{~cm}$ alto, simples a 1-dicotómicas; tallo 10-15 mm ancho (incluyendo las hojas), casi del mismo ancho en toda su extensión, homofilos; tallos 1-2 mm de diámetro (hasta $4 \mathrm{~mm}$ con esporangios), crestados por las bases decurrentes de las hojas; hojas (3-) 5-8 × 0,7-1 mm, dispuestas en verticilos irregulares alternos de 6-7, linear-subuladas, más anchas justo por encima de la base, levemente ascendentes a patentes (a veces reflexas cuando secas), rectas a levemente recurvadas, herbáceas a cartáceas, haz cóncavo cerca de la base y

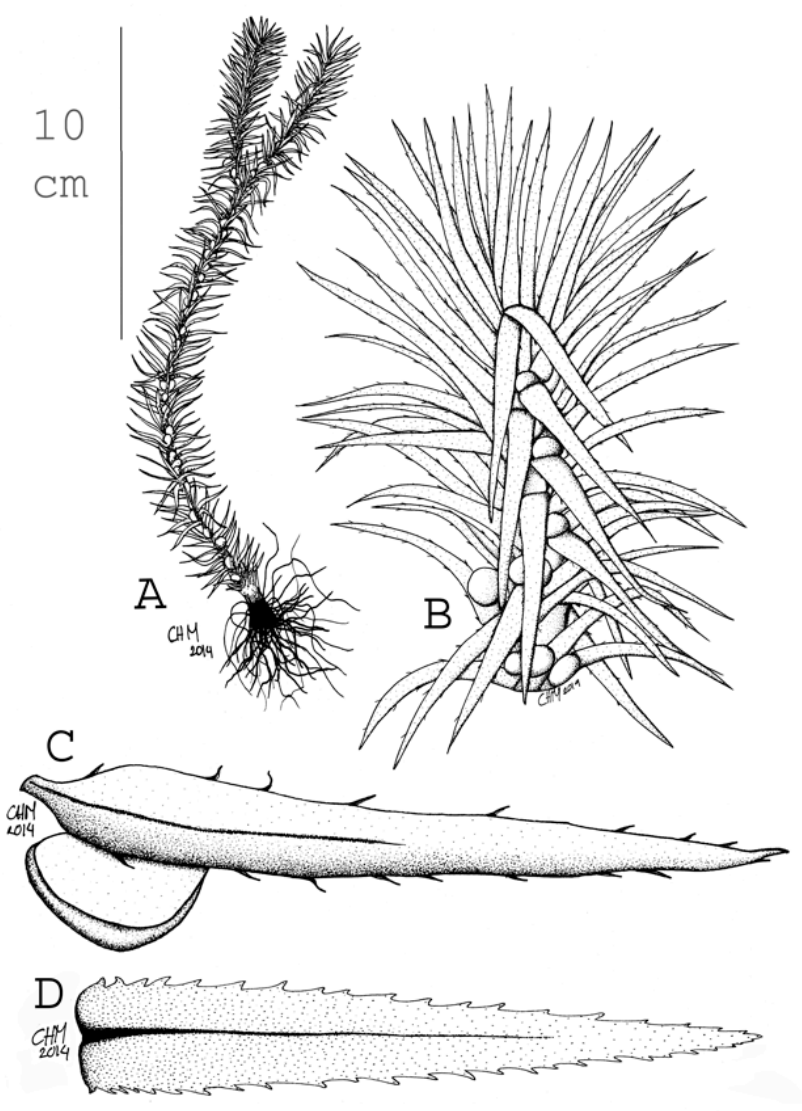

Figura 2: Dos especies parecidas de Phlegmariurus: A-C. P. nanus (A. Rojas \& M. Obando 10517, CR). A) Hábito. B) Detalle de la planta. C) Hoja. D) Hoja de Phlegmariurus reflexus (W. Haber \& W. Zuchowski 9074, CR). Figure 2: Two similar species of Phlegmariurus: A-C. P. nanus (A. Rojas \& M. Obando 10517, CR). A) Habit. B) Plant detail. C) Leaf. D) Leaf of Phlegmariurus reflexus (W. Haber \& W. Zuchowski 9074, CR).

plano o convexo en la parte media y ápice, envés aplanado o escasamente cóncavo a convexo, con una nervadura inconspicua, pálida abaxialmente (al menos en la base), márgenes aplanados a levemente revolutos, esparcidamente ciliados (más densamente en la base), cilios 0,1-0,3 mm de largo, hialinos, perpendiculares a ascendentes hasta en ángulo de $45^{\circ}$ con la costa; base de las hojas a menudo decurrente, ápice largamente agudo; esporangios 1-2 mm de ancho.

Distribución. Costa Rica, conocida solo de la Cordillera Central, bosques nubosos y estacionales, 1550-2100 m.

Etimología. El epíteto específico hace referencia a los tallos pequeños.

Especímenes adicionales revisados. COSTA RICA. Alajuela: camino entre Ciudad Quesada y Zarcero, 
5 abr 1958, M. Álvarez 40 (CR, 38966); Cinco Esquinas de Carrizal, 800 varas antes del Pueblo, 11 abr 1958, $M$. Álvarez 56 (CR, 38969); camino al Volcán Poás, 1950 m, 22 jun 1958, M. Álvarez 63 (CR, 38971). Cartago: entre Volcán Irazú y Hotel Robert, 18 jun 1958, R. Rodríguez \& A. Jiménez 158 (CR, 37060). Heredia: Camino al Volcán Barva, 1990 m, 23 sep 1958, M. Álvarez 113(CR, 38987); ibídem, 1900 m, 23 sep 1958, M. Álvarez 117 (CR, 38990); Santa Bárbara, Santo Domingo, subiendo de Cinco Esquinas, lado Oeste de Volcán Barva, orilla de calle de tierra, $10^{\circ} 07^{\prime} 20 » \mathrm{~N}, 84^{\circ} 06^{\prime} 45$ »W, ca. $2100 \mathrm{~m}$, 3 dic 2014, A. Rojas \& I. Chinchilla 10878 (CR, USJ); San Rafael, Los Ángeles, camino a Cerro Chompipe, $10^{\circ} 04^{\prime} 45^{\prime} \gg \mathrm{N}, 84^{\circ} 04^{\prime} 37 » \mathrm{~W}, 2000 \mathrm{~m}, 28$ set 2013 , A. Rojas et al. 10516 (CR). San José: Coronado, Cascajal, finca de Mario Echandi, $10^{\circ} 00^{\prime} 47^{\prime \prime} \mathrm{N}, 8^{\circ} 57^{\prime} 09^{\prime \prime} \mathrm{W}, 1730$ m, 26 oct 2014, M. Pérez 103 (USJ); camino a Cascajal de Coronado, 28 jul 1957, A. Jiménez 26 (CR); San Ramón de tres Ríos, 20 feb 1959, R. Rodríguez 597 (CR, 36708).

\section{Discusión}

Phlegmariurus gracilis difiere de $P$. reflexus por hojas en verticilos alternos de 4 ó 5 [vs. (6-)7-8 (-9)], éstas más anchas $[0,8-1,5 \mathrm{~mm}$ ancho vs. $0,5-1,0$ $(-1,2) \mathrm{mm}]$, y con el margen entero o disperso y cortamente dentado (vs. densamente dentado (fig. 1).

Øllgaard (1995) citó cinco especímenes: Holm \& Iltis 174, MO; Maxon \& Harvey 8454, UC, US; Davidse et al. 28959, MO; Jiménez 2980, F y Lellinger 1202, US; como un taxón que probablemente amerita reconocimiento taxonómico. Al igual que el espécimen $D$. Lellinger 1202 (CR, US), probablemente los otros especímenes también correspondan a Phlegmariurus gracilis, la especie que se describe aquí.

Phlegmariurus nanus difiere de $P$. reflexus por tallos más cortos [5-11 cm vs. 10-30 $(-40) \mathrm{cm}$ ] y simples a 1-dicotómicos (vs. 2-5-dicotómicos), hojas dispersamente ciliadas ( $v s$. dentadas) y cilios 0,1-0,3 mm (vs. 0,05-0,15 mm largo) (fig. 2).

Phlegmariurus nanus crece en paredones húmedos y márgenes de escorrentía en áreas abiertas, en tanto que $P$. reflexus crece en cualquier paredón o zona medianamente abierta y no tan húmeda, a menudo mezclado con otra vegetación; siendo que esta segunda especie tiene un mayor tamaño, resiste más la competencia con otras plantas.

\section{Agradecimientos}

Este trabajo ha sido posible gracias a la Universidad Nacional de Costa Rica (UNA) y al Sistema Nacional de
Áreas de Conservación (SINAC). También agradezco al Herbario Nacional de Costa Rica (CR) por el préstamo de material y el espacio para revisar los especímenes, a Christian Herrera Martínez por sus excelentes ilustraciones, a Rolando Calderón Fallas por su colaboración en la búsqueda de material, a los revisores anónimos por sus recomendaciones que ayudaron a mejorar este artículo y a todos aquellos que, en una forma o en otra, ayudaron a la realización de este proyecto.

\section{Referencias}

Arana, M. D. y Øllgaard, B. (2012). Revisión de las Lycopodiaceae (Embryopsida, Lycopodiidae) de Argentina y Uruguay. Darwiniana, 50, 266-295.

Holub, J. (1964). Lycopodiella, novy rod radu Lycopodiales. Preslia, 36, 17-21.

Holub, J. (1983). Validation of generic names in Lycopodiaceae: with a description of a new genus Pseudolycopodiella. Folia Geobot. Phytotaxa, 18, 439-442.

Lellinger, D. B. (1989). The ferns and fern-allies from Costa Rica, Panama, and the Chocó. Part I. Pteridologia $2 A$.

Moran, R. C. (2011). Géneros neotropicales de helechos y licófitas, una guía para estudiantes. Organización para estudios tropicales. San José, Costa Rica. 407 p. (Manual fotocopiado).

Øllgaard, B. (1987). A revised classification of the Lycopodiaceae. Opera Botanica, 92, 153-178.

Øllgaard, B. (1988). Lycopodiaceae. In: Harling, G. \& L. Anderson (eds.). Flora of Ecuador, 33, 1-155.

Øllgaard, B. (1992). Neotropical Lycopodiaceae - an overview. Annals of the Missouri Botanical Garden, 79, 687-717.

Øllgaard, B. (1993). Two new Mesoamerican species and a new combination in Huperzia (Lycopodiaceae). Novon, 3, 67-72.

Øllgaard, B. (1995). Lycopodiaceae. In: Moran, R.C. \& R. Riba (eds.). Flora Mesoamericana. Vol. 1. Psilotaceae a Salviniaceae. México Distrito Federal: Universidad Nacional Autónoma de México. p. 5-22.

Øllgaard, B. (2012a). Short communication: Nomenclatural changes in Brazilian Lycopodiaceae. Rodriguésia, 63, 479-482.

Øllgaard, B. (2012b). New combinations in Neotropical Lycopodiaceae. Phytotaxa, 57, 10-22.

Rojas, A. F. (1996). Aportes a la Flora Pteridophyta Costarricense. I. Informes. Brenesia, 45-46, 1-6.

Rojas, A. F. (2005). Novedades en Huperzia Berhh. (Lycopodiaceae) de Costa Rica. Lankesteriana, 5, 109-113.

Warner, W. H. \& Beitel, V. (1992). Generic classification of modern north American Lycopodiaceae. Annals of the Missouri Botanical Garden, 79, 676-686. 